\title{
An Improved Ant Colony Algorithm for Solving the Path Planning Problem of the Omnidirectional Mobile Vehicle
}

\author{
Jiang Zhao, Dingding Cheng, and Chongqing Hao \\ School of Electrical Engineering, Hebei University of Science and Technology, Hebei, Shijiazhuang 050018, China \\ Correspondence should be addressed to Dingding Cheng; chengdingding0306@126.com
}

Received 20 May 2016; Accepted 21 August 2016

Academic Editor: Cheng-Tang Wu

Copyright ( 2016 Jiang Zhao et al. This is an open access article distributed under the Creative Commons Attribution License, which permits unrestricted use, distribution, and reproduction in any medium, provided the original work is properly cited.

\begin{abstract}
This paper presents an improved ant colony algorithm for the path planning of the omnidirectional mobile vehicle. The purpose of the improved ant colony algorithm is to design an appropriate route to connect the starting point and ending point of the environment with obstacles. Ant colony algorithm, which is used to solve the path planning problem, is improved according to the characteristics of the omnidirectional mobile vehicle. And in the improved algorithm, the nonuniform distribution of the initial pheromone and the selection strategy with direction play a very positive role in the path search. The coverage and updating strategy of pheromone is introduced to avoid repeated search reducing the effect of the number of ants on the performance of the algorithm. In addition, the pheromone evaporation coefficient is segmented and adjusted, which can effectively balance the convergence speed and search ability. Finally, this paper provides a theoretical basis for the improved ant colony algorithm by strict mathematical derivation, and some numerical simulations are also given to illustrate the effectiveness of the theoretical results.
\end{abstract}

\section{Introduction}

Path planning, which is one of the hot topics in motion control research, requires the control object to determine the path, avoid obstacles, and achieve the goal autonomously [1]. As a special kind of mobile robot, the omnidirectional mobile robot has wide application in the field of industrial transportation. With the increase of production cost and work efficiency, the path planning problem of the omnidirectional mobile vehicle needs to be resolved as soon as possible. The path planning problem of mobile robot was proposed in the 1960s. With the research of Lozano-Perez and Wesley [2], the problem has aroused the interest of many scholars.

Simulated annealing algorithm [3], potential function theory [4], genetic algorithm [5], particle swarm algorithm [6], and ant colony algorithm [7] are representative methods of path planning, which can find the shortest path. In addition, the research on path planning has made great progress in recent years. The idea of $\mathrm{Mo}$ and $\mathrm{Xu}$ [8] solved the path planning by PSO with the position updating strategy and biogeography particle swarm optimization algorithm to increase the diversity of population and optimize the paths in a static environment. Precup et al. [9] proposed that the adaptive charged system search algorithm was applied to the optimal path planning problem of multiple mobile robots in static environment. Several modifications and improvements of A star algorithm were introduced by Ducho et al. [10] and Guruji et al. [11] considering a static or dynamic environment. And it was the first time that MOVNS [12] was proposed to deal with the path planning problem of mobile robots about the path safety, length, and smoothness. In addition, many other findings also made contributions to the related path planning problem [13-15].

Many scholars applied ant colony algorithm [16] to solve the path planning problem because it can represent obstacles flexibly and easily by using a tabu list. On this basis, the further research was carried out. Later, according to the characteristics of the above algorithms, some methods combined ant colony algorithm and other algorithms which were proposed and had more advantages than one algorithm, such as ant colony particle swarm algorithm [2] and ant colony genetic algorithm [17]. However, the application of ant colony algorithm is much more than that. As discussed by Tiwari and Vidyarthi [18], the ant colony optimization algorithm solved 




FIGURE 1: The omnidirectional mobile vehicle.

the scheduling problem effectively through a kind of special ants that remain alive till the fitter lazy ants are generated in the successive generations. Wang et al. [19] proposed a modified ACS scheme that can identify the true attack path even without the entire network routing information. Jiang et al. [20] presented a coevolutionary improved multiant colony optimization algorithm to design appropriate pipe routes in the layout space under various kinds of constraints. What is more, Saidi-Mehrabad et al. [21] also made some improvements to the ant colony algorithm to solve the problem. Therefore, solving the problem in accordance with the feature of the control object, environment, and existing methods is the best way.

So, accordingly, ant colony algorithm is a key point, because it is the basis of the above methods. Therefore, based on the motion characteristics of omnidirectional mobile vehicle, this paper proposes a kind of improved ant colony algorithm. The initial pheromone distribution is nonuniform, which helps reduce the blindness of the search. In the strategy of selection, heuristic information with direction is introduced to improve the probability of finding the optimal solution. And piecewise adjusting pheromone behavior helps avoid falling into local optimal solution. Finally, the pheromone coverage and updating strategy can ensure that the algorithm can find the optimal path strictly in theory.

\section{Problem Statement}

2.1. Control Object. As we all know, solving the path planning problem in accordance with the feature of the control object is the best way. Different from mobile robot, the omnidirectional mobile vehicle, as shown in Figure 1, is equipped with chassis and drive which can contribute to moving in any direction. In terms of its control system, the omnidirectional mobile vehicle has 8 basic motion units, upper, upper right, right, lower right, lower, lower left, left, and upper left, as shown in Figure 2. Therefore, its form of motion, which is the output of the motion path, can be composed of a number of basic motion units.

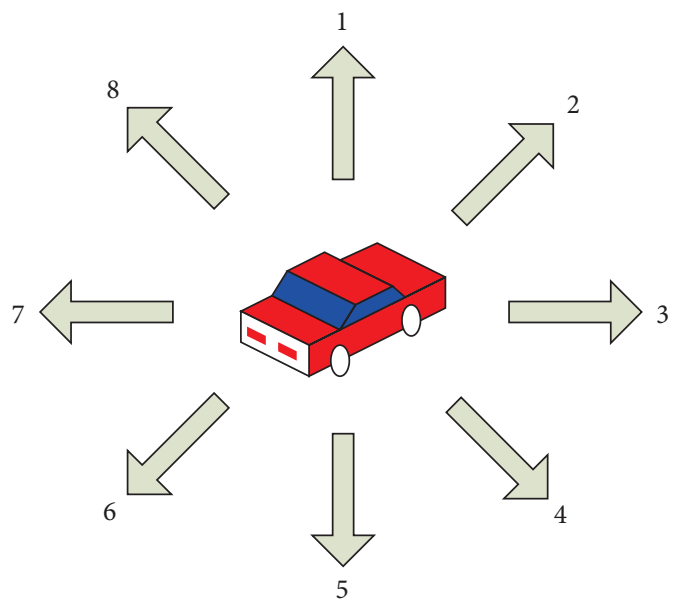

Figure 2: Basic motion directions.

2.2. Problem Description. Without considering the omnidirectional mobile vehicle height, the working environment can be considered as a two-dimensional plane. So the path planning needs to solve three problems:

(1) In a nonchanging work environment, find a path to connect the starting point and the ending point.

(2) Find the shortest path with avoiding all obstacles.

(3) The algorithm should have certain simplicity, low complexity, and good stability.

\section{Mathematical Model}

3.1. Environment Model. In practical applications, the electronic map is often needed to represent the working environment. This paper describes the work environment using the grid method in which the grid coordinates are replaced by the position of each center point. So working environment is divided into $M * N$ squares. And the obstacles are represented by gray grids (less than one by one count) distinguishing them from available parts. The area is numbered from top to bottom and from left to right in the literature [22], while the squares can be represented by the set of numbered $B=$ $\{1,2, \ldots, b, \ldots, M * N\}$ which is shown in Figure 3. In order to identify obstacles, the white grid cell is represented by 0 and the gray grid unit is represented by 1 . Thus, the working environment is represented by $M * N$ binary matrix, which is denoted by $G$.

3.2. Path Representation. It can be known from the characteristics of the omnidirectional mobile vehicle and the environment representation that the resolution of the system is related to the number of grids. Finally, the path is obtained by adding the components of the solution step by step to obtain the solution. The path of the vehicle can be expressed as $L=\left\{\left(x_{1}, y_{1}\right), \ldots,\left(x_{i}, y_{i}\right), \ldots,\left(x_{t-1}, y_{t-1}\right),\left(x_{t}, y_{t}\right)\right\}$, the starting point is $\left(x_{1}, y_{1}\right)$ denoted as $S$, and the ending point coordinate is $\left(x_{t}, y_{t}\right)$ denoted as $E$. From the environmental model, we can know that the path can be expressed by the 


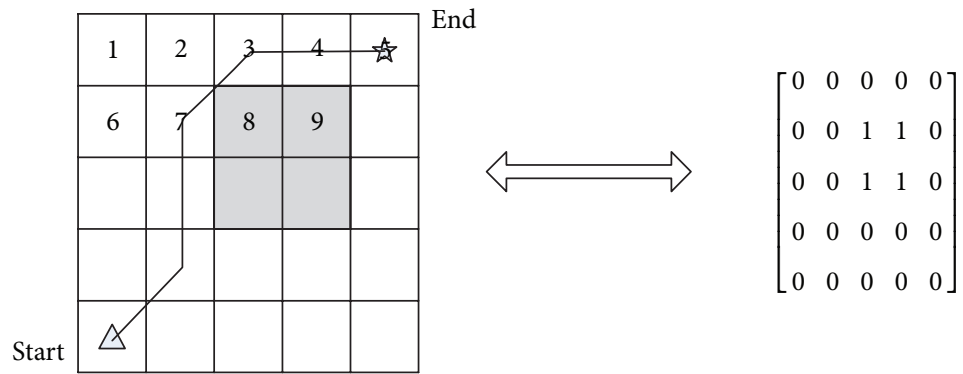

FIgURE 3: Mathematical model of working environment.

grid number $L=\left\{l_{1}, \ldots, l_{i}, \ldots, l_{t-1}, l_{t}\right\}$ and $\left(x_{i}, y_{i}\right)$ is replaced by $l_{i}, i=1, \ldots, t-1, t$.

\section{Improved Ant Colony Algorithm}

\subsection{Improvement Aspects}

(1) Nonuniform Distribution of Initial Pheromone. In the initial phase of traditional ant colony algorithm, the pheromone distribution is described by $M * N$ dimensional matrix, where the element $\tau_{b} \in T$ represents the initial value of pheromone in $b$ grid. As shown below,

$$
\tau_{b}=c_{0} \quad c_{0} \text { is a constant. }
$$

To solve the problems of lack of initial pheromone and low speed of search, in literatures $[10,11]$ particle swarm optimization algorithm and genetic algorithm are proposed to generate an initial path which can be transformed into the initial pheromone distribution so as to reduce the blindness of the ant colony search. In order to improve the efficiency and simplicity of the algorithm, the nonuniform distribution of initial pheromone is proposed in this paper. So the pheromone distribution is described by matrix $T$, where element $\tau_{b} \in T$, as shown below:

$$
\tau_{b}= \begin{cases}c_{0} \times \theta_{1} & b \in A \\ c_{0} & \text { otherwise }\end{cases}
$$

where $A$ is the collection of all numbers between number $l_{1}$ and $l_{t}$; that is to say, $A=\left\{\min \left\{l_{1}, l_{t}\right\}, \min \left\{l_{1}, l_{t}\right\}+\right.$ $\left.1, \ldots, \max \left\{l_{1}, l_{t}\right\}\right\} ; \theta_{1}$ is a constant greater than 1 . Formula (2) indicates that the number of pieces of information between the starting point and the ending point is slightly higher than others. Similar to the principle of the zero point theorem, in view of the characteristics of the path, the probability of an arbitrary path set between the starting point and the ending point is 1 . Therefore, the simple initial distribution strategy has advantages to reduce the blindness of ant colony search, shorten the path search time. And at the same time, it does not increase the complexity of the algorithm.
(2) Heuristic Strategy with Direction Information. In the traditional ant colony algorithm, the probability of the next node is selected by rotating the roulette wheel method as follows:

$$
\begin{aligned}
& p_{l_{i}, l_{i+1}}=\frac{\left(\tau_{l_{i}, l_{i+1}}\right)^{\alpha}\left(\eta_{l_{i}, l_{i+1}}\right)^{\beta}}{\sum_{l_{i+1} \in \operatorname{allowed}(b)}\left(\tau_{l_{i}, l_{i+1}}\right)^{\alpha}\left(\eta_{l_{i}, l_{i+1}}\right)^{\beta}}, \\
& \eta_{l_{i}, l_{i+1}}=\frac{1}{d_{l_{i+1}, l_{t}}}, \\
& d_{l_{i+1}, l_{t}}=\sqrt{\left(x_{l_{t}}-x_{l_{i+1}}\right)^{2}+\left(y_{l_{t}}-y_{l_{i+1}}\right)^{2}},
\end{aligned}
$$

where $p_{l_{i}, l_{i+1}}$ is the probability of the next node of $l_{i}, \tau_{l_{i}}, l_{i+1}$ is the pheromone of the path from $l_{i}$ to $l_{i+1}$, and $\alpha$ is the pheromone coefficient. Even $\eta_{l_{i}, l_{i+1}}$ is the heuristic information of $l_{i}$ to $l_{i+1}$, and $\beta$ is the heuristic information parameter, where $d_{l_{i+1}, l_{t}}$ is the distance between node $l_{i+1}$ and end node $l_{t}$. $\left(x_{l_{i+1}}, y_{l_{i+1}}\right)$ and $\left(x_{l_{t}}, y_{l_{t}}\right)$ are the coordinates of $l_{i+1}$ and $l_{t}$.

The direction information is proposed as the heuristic information of the node transfer strategy by Wang et al. [19] to solve the path planning problem without obstacle:

$$
\begin{aligned}
& p_{l_{i}, l_{i+1}}=\frac{\left(\tau_{l_{i}, l_{i+1}}\right)^{\alpha}\left(\eta_{l_{i}, l_{i+1}}\right)^{\beta}}{\sum_{l_{i+1} \in \operatorname{allowed}(b)}\left(\tau_{l_{i}, l_{i+1}}\right)^{\alpha}\left(\eta_{l_{i}, l_{i+1}}\right)^{\beta}}, \\
& \eta_{l_{i}, l_{i+1}}= \begin{cases}\theta_{2} & \text { if state is toward the goal } \\
1 & \text { otherwise, }\end{cases}
\end{aligned}
$$

where $\eta_{l_{i}, l_{i+1}}$ is the direction of information and $\theta_{2}$ is a constant little more than 1 . In order to reduce the blindness of the search, the paper puts forward the strategy of the direction information, and the probability formula is

$$
\begin{aligned}
& p_{l_{i}, l_{i+1}} \\
& = \begin{cases}\frac{\left(\tau_{l_{i}, l_{i+1}}\right)^{\alpha}\left(\eta_{l_{i}, l_{i+1}}\right)^{\beta} \mu_{l_{i}, l_{i+1}}}{\sum_{l_{i+1} \in \text { allowed }(b)}\left(\tau_{l_{i}, l_{i+1}}\right)^{\alpha}\left(\eta_{l_{i}, l_{i+1}}\right)^{\beta} \mu_{l_{i}, l_{i+1}}} & l_{i+1} \in \text { the next node } \\
0 & \text { otherwise, }\end{cases}
\end{aligned}
$$




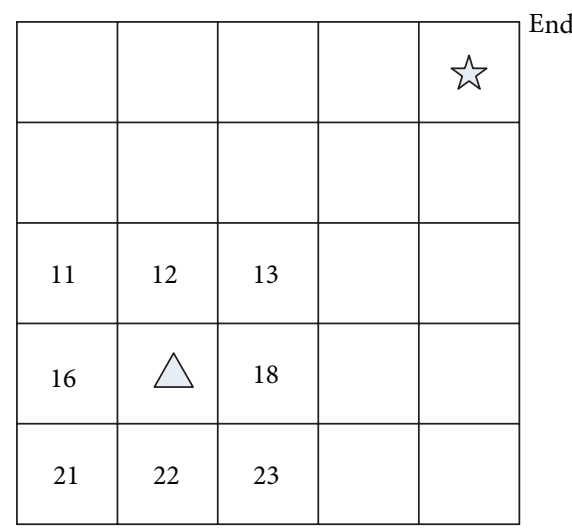

FIGURE 4: Directional information representation.

where

$$
\begin{aligned}
& \eta_{l_{i}, l_{i+1}}=\frac{1}{d_{l_{i+1}, l_{t}}} \\
& d_{l_{i+1}, l_{t}}=\sqrt{\left(x_{l_{t}}-x_{l_{i+1}}\right)^{2}+\left(y_{l_{t}}-y_{l_{i+1}}\right)^{2}} .
\end{aligned}
$$

The direction information $\mu_{i, i+1}$ is expressed as follows:

$$
\mu_{l_{i}, l_{i+1}}=\theta_{3}^{\gamma},
$$

where $\gamma$ is the number of the same directions of node $l_{i}$ to next $l_{i+1}$ and node $l_{i}$ to end $l_{t}: \gamma=0,1,2$. And $\theta_{3}$ is a constant slightly greater than 1 . As shown in Figure 4 , the next node of 17 may be $11,12,13,16,18,21,22$, and 23 , where node 13 has two same directions as the end, so the direction information is $\theta_{3}^{2}$. And as such, the direction information of nodes 11,12 , 18 , and 23 is $\theta_{3}^{1}$, and for 16,21 , and 22 it is $\theta_{2}^{0}$.

(3) Coverage and Updating Strategy. In the traditional ant colony algorithm, the next node position is decided by the roulette wheel method and repeated until the target point is obtained. The pheromones of all nodes are updated by the following rules after every ant $\xi$ searching:

$$
\begin{aligned}
\tau_{l_{i}, l_{i+1}}(k, \xi+1) & =(1-\rho) \tau_{l_{i}, l_{i+1}}(k, \xi)+\Delta \tau_{l_{i}, l_{i+1}}(k, \xi), \\
\tau_{l_{i}, l_{i+1}}(k+1,1) & =(1-\rho) \tau_{l_{i}, l_{i+1}}(k, R)+\Delta \tau_{l_{i}, l_{i+1}}(k, R), \\
\Delta \tau_{l_{i}, l_{i+1}}(k, \xi) & = \begin{cases}\frac{1}{\mathrm{PL}_{k, \xi}} & \text { through } l_{i} \text { to } l_{i+1} \\
0 & \text { otherwise, }\end{cases}
\end{aligned}
$$

where $\rho$ is the evaporation rate of pheromone, where the function is to avoid the pheromone accumulation: $0<\rho<1$. $R$ is the number of the ants starting from the starting point in every round. $\Delta \tau_{l_{i}, l_{i+1}}(k, \xi)$ is the addition of the $k$ th round $\xi$ th ant. And $\mathrm{PL}_{k, \xi}$ is the path length of the $\xi$ th ant which can get to the end point in the $k$ th round.

In the improved ant colony algorithm, the next node position is decided by the roulette wheel method until the target point is obtained. And, in a cycle, the pheromone of all the nodes is updated by following the rules in each round of ants:

$$
\begin{aligned}
& \tau_{l_{i}, l_{i+1}}^{\prime}(k+1)=(1-\rho(k)) \tau_{l_{i}, l_{i+1}}(k)+\Delta \tau_{l_{i}, l_{i+1}}(k), \\
& \tau_{l_{i}, l_{i+1}}^{\prime \prime}(k+1)=\frac{\theta_{4} \tau_{l_{i}, l_{i+1}}(0)}{\ln (k+1)}+\Delta \tau_{l_{i}, l_{i+1}}(k), \\
& \tau_{l_{i}, l_{i+1}}(k+1)=\max \left\{\tau_{l_{i}, l_{i+1}}^{\prime}(k+1), \tau_{l_{i}, l_{i+1}}^{\prime \prime}(k+1)\right\} .
\end{aligned}
$$

Among them, $\Delta \tau_{l_{i} l_{i+1}}(k)$ is the pheromone update part of $l_{i}$ to $l_{i+1}$ in the $k$ th round. $\theta_{4}$ is a constant, and the maximum value of the pheromone in the iterative process is the initial set value $\tau_{\max }=\tau(0) . \rho(k)$ is the pheromone evaporation coefficient: $0<\rho(k)<1$. In the entire search space,

$$
\begin{gathered}
\Delta \tau_{l_{i}, l_{i+1}}(k)=o\left(\Delta \tau_{l_{i}, l_{i+1}}(k, 1), \ldots, \Delta \tau_{l_{i}, l_{i+1}}(k, \xi), \ldots,\right. \\
\left.\Delta \tau_{l_{i}, l_{i+1}}(k, R-1), \Delta \tau_{l_{i}, l_{i+1}}(k, R)\right),
\end{gathered}
$$

where $o\left(\Delta \tau_{l_{i}, l_{i+1}}(k, 1), \ldots, \Delta \tau_{l_{i}, l_{i+1}}(k, R)\right)$ is a nonzero pheromone space covering operation. For example,

$$
\begin{aligned}
A(1) & =\left[\begin{array}{lll}
1 & 2 & 4 \\
0 & 0 & 1 \\
3 & 0 & 0
\end{array}\right], \\
A(2) & =\left[\begin{array}{lll}
2 & 3 & 5 \\
1 & 0 & 0 \\
2 & 0 & 0
\end{array}\right], \\
\text { so } o(A(1), A(2)) & =\left[\begin{array}{lll}
2 & 3 & 5 \\
1 & 0 & 1 \\
2 & 0 & 0
\end{array}\right] .
\end{aligned}
$$

In formula (10),

$$
\Delta \tau_{l_{i}, l_{i+1}}(k, \xi)= \begin{cases}\frac{Q}{\mathrm{PL}_{k, \xi}} \tau_{l_{i}, l_{i+1}}(k, \xi) & \text { through } l_{i} \text { to } l_{i+1} \\ 0 & \text { otherwise. }\end{cases}
$$

Among them, $Q$ is a constant. It can be seen that if the ant can get to the end point, the added part of the pheromone is in inverse proportion with the length and if the ant cannot reach the ending point, it is recorded as 0 . The rule can guide the search for the shortest path, improve the speed of convergence, and avoid the possibility of the pheromone accumulation caused by repeated search; meanwhile the effect on the performance of the algorithm caused by the quantity of the ants is reduced.

(4) Evaporation Coefficient Segment. When the problem scale is relatively large, due to the presence of pheromone evaporation, the pheromone of some nodes will be reduced greatly, even close to zero, which reduces the search ability of the algorithm. When $\rho$ is large, the search ability is affected 
by repeated selection. Meanwhile when $\rho$ is small, the search ability of the algorithm is enhanced, but the convergence speed is decreased. Therefore, the heuristic information coefficient is adjusted as follows:

$$
\rho(k+1)= \begin{cases}\theta_{5} \rho(k) & \rho(k) \geq \rho_{\min } \\ \rho_{\min } & \rho(k)<\rho_{\min }\end{cases}
$$

where $\theta_{5}$ is a constant less than $1 . k$ is the number of search rounds: $k=0,1, \ldots, K \cdot \rho(0)=\rho_{\max } \cdot \rho_{\max }$ and $\rho_{\min }$ are the maximum and minimum of the coefficient of evaporation. The pheromone evaporation coefficient of fragmentation can enhance the search ability in the initial stage of search, increase the convergence speed later, and improve the performance of ant colony algorithm.

4.2. Algorithm Steps. The improved ant colony algorithm is to find the optimal path according to the following steps.

Step 1. The nonuniform distribution of initial pheromone is proposed and pheromone matrix $T$ is constructed according to formula (2).

Step 2. Send $K$ rounds and each round of $R$ ants which are placed at the starting point.

Step 3. Send a round of ants and each ant to select the node according to the roulette wheel method, with the probability of each point calculated by formulas (5), (6), and (7).

Step 4. After a round of ant search, the pheromone was calculated by formulas (10) and (12), the evaporation coefficient was calculated by (13), and the pheromone was updated according to formulas (9).

Step 5. Send the next round of ants, and repeat above Steps 3 and 4 until the end of the iteration.

Step 6. Record the shortest path of each round; then draw the curve of the shortest path length of each round.

Step 7. Compare the shortest paths of all the ants. Finally, output the current global optimal path.

4.3. Proof of Convergence. The proof depends on a necessary assumption: there is a path between the starting point and the ending at least. According to formulas (10) and (12), the added part of pheromone must be greater than or equal to 0 . That is,

$$
\Delta \tau_{l_{i}, l_{i+1}}(k) \geq 0
$$

Formulas (9) show that the pheromone can be expressed as follows:

$$
\tau_{l_{i}, l_{i+1}}(k) \geq \max \left((1-\rho(k))^{k} \tau(0), \frac{\theta_{4} \tau(0)}{\ln (k+1)}\right) .
$$

Event $E_{k}$ means that the optimal solution is obtained for the first time in the $k$ th iteration. Therefore, event $\bigwedge_{k=1}^{K} E_{k}$ denotes that the algorithm can find the optimal solution for the first time for $K$ iterations. Then probability $P\left(\bigwedge_{k=1}^{K} E_{k}\right)$ is satisfied:

$$
\lim _{k \rightarrow \infty} P\left(\bigwedge_{k=1}^{K} E_{k}\right)=1
$$

Proof. $l_{i+1}^{*}$ is the choice of the $i$ th optimal solution, and the probability of finding the optimal solution for the $\xi$ th ant in the $k$ th round $p(k, \xi)$ can be obtained by formula (5) because the node selection is an independent event.

$$
\begin{aligned}
& 1 \geq p(k, \xi)=\prod_{i=1}^{t-1} \frac{\left(\tau_{l_{i}, l_{i+1}^{*}}\right)^{\alpha}\left(\eta_{l_{i}, l_{i+1}^{*}}\right)^{\beta} \mu_{l_{i}, l_{i+1}^{*}}}{\sum_{l_{i+1} \in \operatorname{allowed}(b)}\left(\tau_{l_{i}, l_{i+1}}\right)^{\alpha}\left(\eta_{l_{i}, l_{i+1}}\right)^{\beta} \mu_{l_{i}, l_{i+1}}} \\
& =\prod_{i=1}^{t-1} \frac{\left(\tau_{l_{i}, l_{i+1}^{*}}\right)^{\alpha}}{\sum_{l_{i+1} \in \operatorname{allowed}(b)}\left(\tau_{l_{i}, l_{i+1}}\right)^{\alpha}\left(\eta_{l_{i}, l_{i+1}} / \eta_{l_{i}, l_{i+1}^{*}}\right)^{\beta}\left(\mu_{l_{i}, l_{i+1}} / \mu_{l_{i}, l_{i+1}^{*}}\right)} .
\end{aligned}
$$

Define $\psi\left(l_{i+1}\right)=\left(\eta_{l_{i}, l_{i+1}} / \eta_{l_{i}, l_{i+1}^{*}}\right)^{\beta}\left(\mu_{l_{i}, l_{i+1}} / \mu_{l_{i}, l_{i+1}^{*}}\right)$, and $\psi_{\max }=$ $\max \left\{\psi\left(l_{i+1}\right)\right\}, i=1, \ldots, t-1$. So formula (17) can be expressed as

$$
\prod_{i=1}^{t-1} \frac{\left(\tau_{l_{i}, l_{i+1}^{*}}\right)^{\alpha}}{\sum_{l_{i+1} \in \operatorname{allowed}(b)}\left(\tau_{l_{i}, l_{i+1}}\right)^{\alpha} \psi_{\max }} .
$$

Because of formula (12), it is known that the minimum value of pheromone in the $k$ th iteration of the ant colony algorithm is

$$
\tau_{\min }(k) \geq \frac{\theta_{4} \tau(0)}{\ln (k+1)}, \quad \forall k \geq 1
$$

Further, the maximum value of the pheromone is the initial value by setting some parameters. So

$$
\tau_{\max }=\tau(0)
$$

And the maximum number of $N_{c}\left(k, \xi,\left(l_{i}, l_{i+1}\right)\right)$ can be expressed as

$$
N_{c}=\max \left\{N_{c}\left(k, \zeta,\left(l_{i}, l_{i+1}\right)\right)\right\}, \quad i=1, \ldots, t-1,
$$

where $N_{c}\left(k, \xi,\left(l_{i}, l_{i+1}\right)\right)$ is the number of options. By (19), (20), and (21), we can know that formula (18) meets the following relationship:

$$
\begin{aligned}
1 & \geq P(k, \zeta)>\prod_{i=1}^{t-1} \frac{\left(\tau_{l_{i}, l_{i+1}^{*}}\right)^{\alpha}}{\sum_{l_{i+1} \in \operatorname{allowed}(b)}\left(\tau_{l_{i}, l_{i+1}}\right)^{\alpha} \psi_{\max }} \\
& >\left(\frac{\left(\theta_{4} \times \tau(0) / \ln (k+1)\right)^{\alpha}}{(\tau(0))^{\alpha} \times N_{c} \times \psi_{\max }}\right)^{t-1} .
\end{aligned}
$$


Record

$$
\begin{aligned}
d & =\left(\frac{\left(\theta_{4} \times \tau(0)\right)^{\alpha}}{(\tau(0))^{\alpha} \times N_{c}\left(k, \xi,\left(l_{i}, l_{i+1}\right)\right) \times \psi_{\max }}\right)^{t-1} \\
& =\left(\frac{\left(\theta_{4}\right)^{\alpha}}{N_{c}\left(k, \xi,\left(l_{i}, l_{i+1}\right)\right) \times \psi_{\max }}\right)^{t-1} .
\end{aligned}
$$

So, formula (22) is expressed as

$$
\begin{aligned}
1 & \geq P(k, \zeta)>\prod_{i=1}^{t-1} \frac{\left(\theta_{4} \times \tau(0) / \ln (k+1)\right)^{\alpha}}{(\tau(0))^{\alpha} \times N_{c} \times \psi_{\max }} \\
& =\frac{d}{(\ln (k+1))^{(t-1) \alpha}} .
\end{aligned}
$$

From the above, we can know that

$$
1>\frac{d}{(\ln (k+1))^{(t-1) \alpha}}>0 .
$$

The probability that the optimal solution cannot be found by any ants in the $k$ th round is $p(k)$ :

$$
p(k)=(1-p(k, \xi))^{R} .
$$

Probability $P\left(\bigvee_{k=1}^{K} \bar{E}_{k}\right)$ that cannot find the optimal solution for the $K$ rounds is given as follows:

$$
\begin{aligned}
P\left(\bigvee_{k=1}^{K} \bar{E}_{k}\right) & =\prod_{k=1}^{K} p(k)=\prod_{k=1}^{K}(1-p(k, \xi))^{R} \\
& <\prod_{k=1}^{K}\left(1-\frac{d}{(\ln (k+1))^{(t-1) \alpha}}\right)^{R} .
\end{aligned}
$$

After the logarithm to the above formula,

$$
\begin{aligned}
& \ln P\left(\bigvee_{k=1}^{K} \bar{E}_{k}\right)<\sum_{k=1}^{K} \ln \left[1-\left(\frac{d}{\ln (k+1)^{(t-1) \alpha}}\right)\right]^{R} \\
& =R \sum_{k=1}^{K} \ln \left[1-\left(\frac{d}{\ln (k+1)^{(t-1) \alpha}}\right)\right] \\
& \leq-R \times d \sum_{k=1}^{K}\left(\frac{1}{\ln (k+1)^{(t-1) \alpha}}\right) \\
& \leq-R \times d \sum_{k=1}^{K} \frac{1}{k+1} \\
& \lim _{K \rightarrow \infty} \ln P\left(\bigvee_{k=1}^{K} \bar{E}_{k}\right)<\lim _{K \rightarrow \infty} \ln \left(-R \times d \sum_{k=1}^{K} \frac{1}{k+1}\right) \\
& \quad=-\infty .
\end{aligned}
$$

That is, $P\left(\bigvee_{k=1}^{K} \bar{E}_{k}\right)=0$. So $\lim _{k \rightarrow \infty} P\left(\bigwedge_{k=1}^{K} E_{k}\right)=1$.

\section{Numerical Simulations}

The experiments are made to demonstrate the effectiveness of the proposed algorithm. The algorithm is compiled in MATLAB software. Experiments were conducted using a comparative method to be more persuasive along with the same experimental conditions.

5.1. Simulation Experiments. The experiment was divided into three parts with two algorithms, the traditional ant colony algorithm and the improved ant colony algorithm. In order to compare the effects of the two algorithms, they are used in the same environment.

(1) Example 1. In this example, set the simulation environment as $20 \times 20$ grids and the length of each unit is 1 . The starting point is the upper left corner in the grid $(0.5,19.5)$, and the end point is the lower right corner $(19.5,0.5)$ (see Figure 5).

(2) Example 2. We set the environment as $30 \times 30$ grids and the length of the unit is 1 . The starting point is $(0.5,8.5)$, and the end point is $(25.5,28.5)$ (see Figure 6).

(3) Example 3. The experiment is made in model of $40 \times 40$ grids and the length of each unit is 1 . The starting point is set at $(0.5,39.5)$, and the ending point is set at $(39.5,0.5)$ (see Figure 7).

5.2. Results Analysis. As shown in Figure 5(a), it is a global optimal path in the case of Example 1. Similarly, Figures $6(\mathrm{a})$ and $7(\mathrm{a})$ are the global optimal paths in Examples 2 and 3 , respectively. The obstacles in each environment are randomly selected. The three experiment results show that the ant colony algorithm and the improved ant colony algorithm both can find the global optimal paths in a variety of environments.

Figures 5(b) and 5(c) depict the path length iteration curves in Example 1 by traditional ant colony algorithm and the improved ant colony algorithm. And the search process about the shortest path length of each round and the current global optimal path length can be seen from the figures. By comparison, it can be seen that the shortest path length is found in the 49th iteration by traditional ant colony algorithm, while it is found in the fifth iteration by the improved algorithm. And once the optimal solution is obtained, the search will converge to the shortest path value by the improved algorithm. Similarly, Figures 6(b) and 6(c) represent the search process of the two algorithms in Example 2. The traditional ant colony algorithm finds the shortest path in the 69th iteration with value of 35.63, while the improved algorithm gets the shortest path in the ninth times. In the same way, Figures $7(\mathrm{~b})$ and $7(\mathrm{c})$ show that the traditional ant colony algorithm and the improved ant colony algorithm find the shortest path in the 37th and 24th iterations, respectively. Therefore, it can be seen that the search efficiency of the improved ant colony algorithm is 


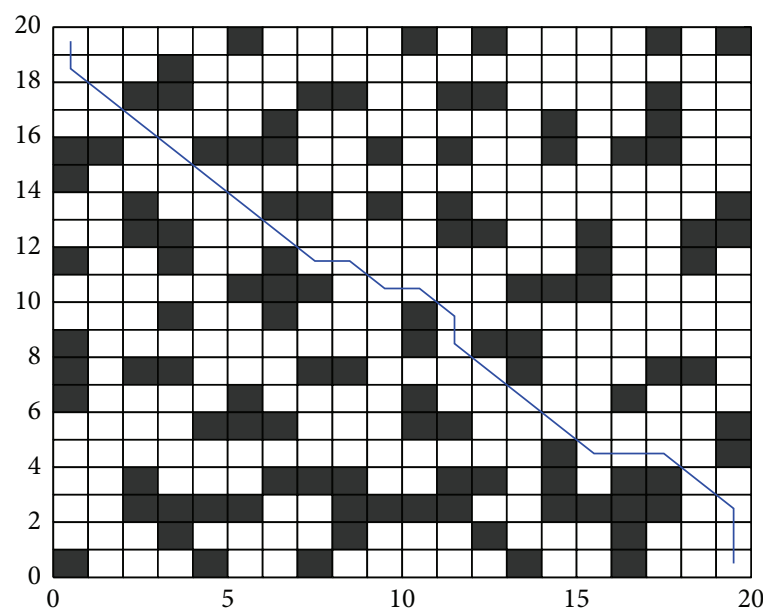

(a)



(c)

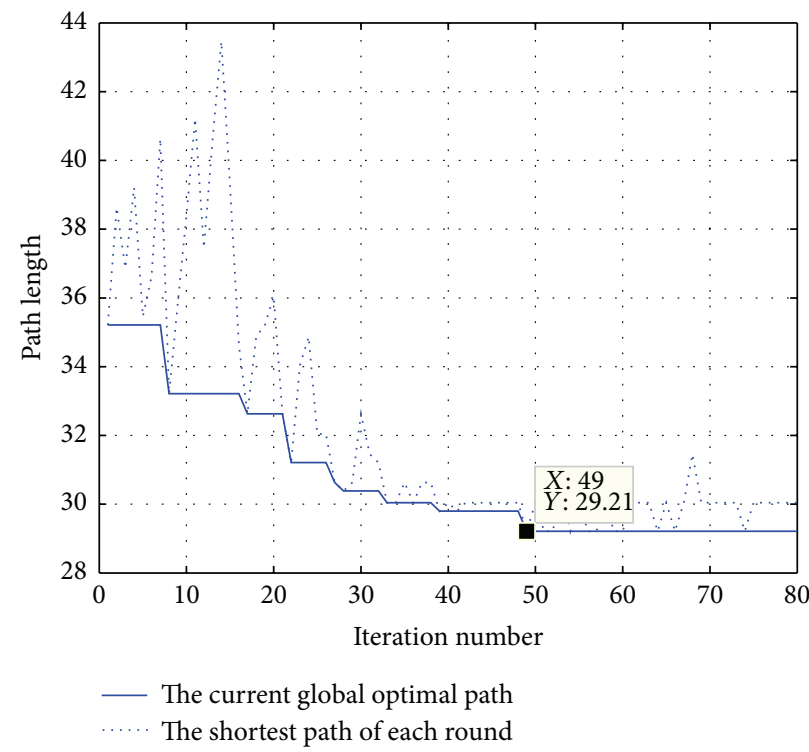

(b)

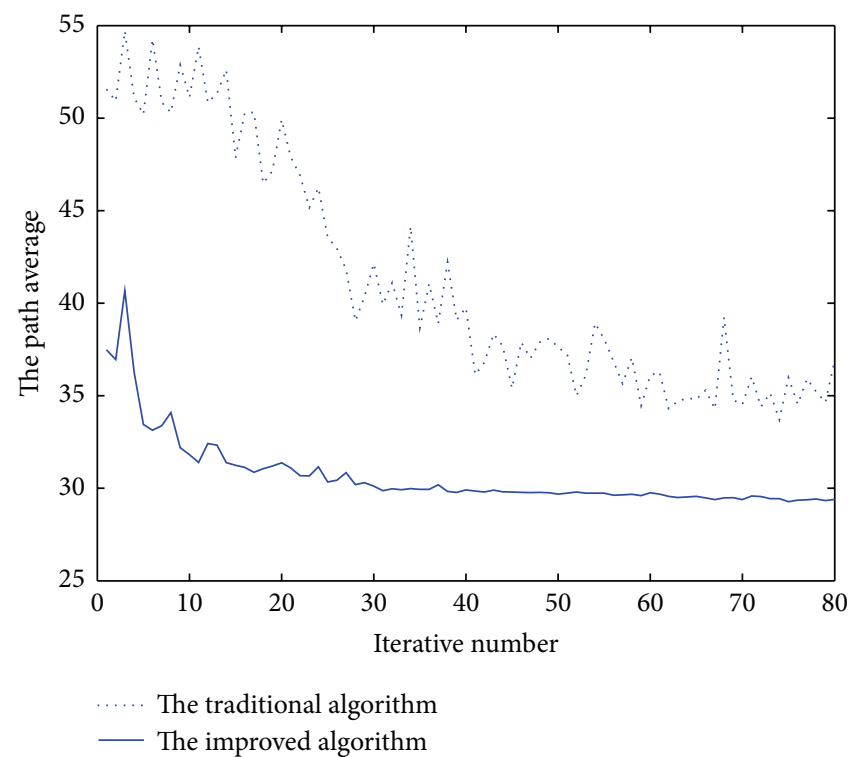

(d)

FIGURE 5: (a) Simulation results in $20 \times 20$ grids. (b) The iterative curve by the traditional algorithm. (c) The iterative curve by the improved algorithm. (d) The path average curve length by two algorithms.

significantly higher compared to the traditional ant colony algorithm in a variety of environment.

The mean value of path iterative length often represents the convergence ability of the algorithm. Figures 5(d), 6(d), and $7(d)$ present the contrast of the two algorithms on the search path average value. The improved algorithm not only is faster than the ant colony algorithm but also can avoid falling into local minimum point and obtain the global optimal solution effectively either in simple environment or in a complex one.

\section{Conclusion}

In this paper, an improved ant colony algorithm is proposed for the 8 control operating units of the omnidirectional mobile vehicle. The grid method is used to establish the environment model, and the tabu list is introduced. By using the tabu list to show the obstacles and the units that have passed through, it is flexible to deal with obstacles and avoid duplicating the path. In this paper, the initial distribution of nonuniformity pheromone is presented which improves 


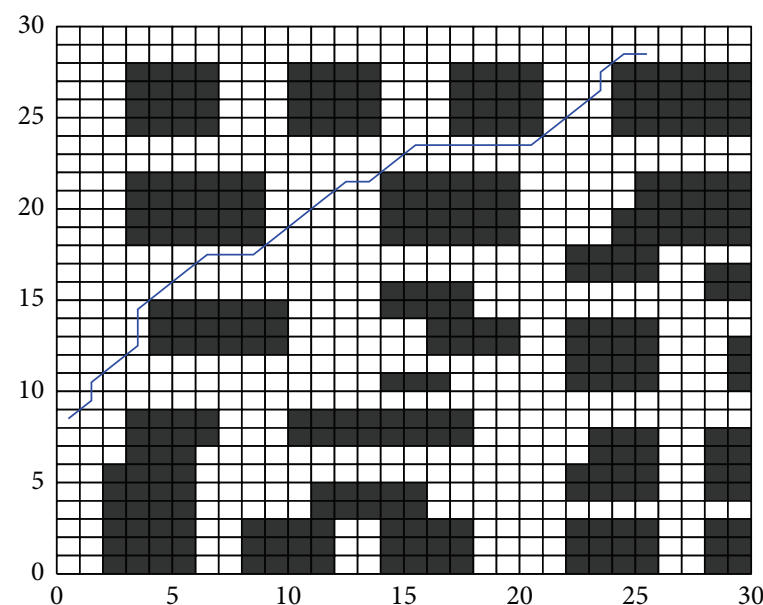

(a)

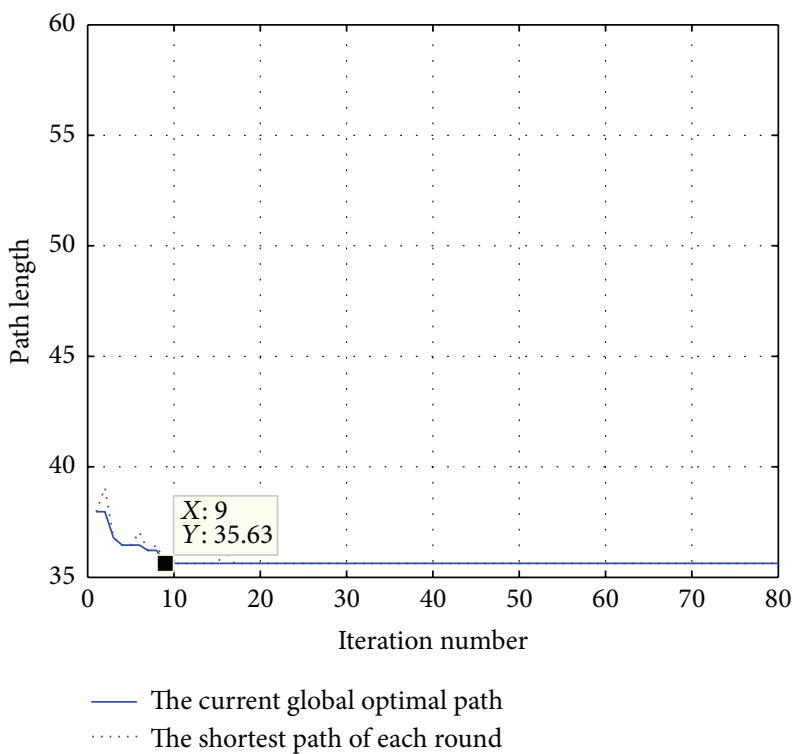

(c)

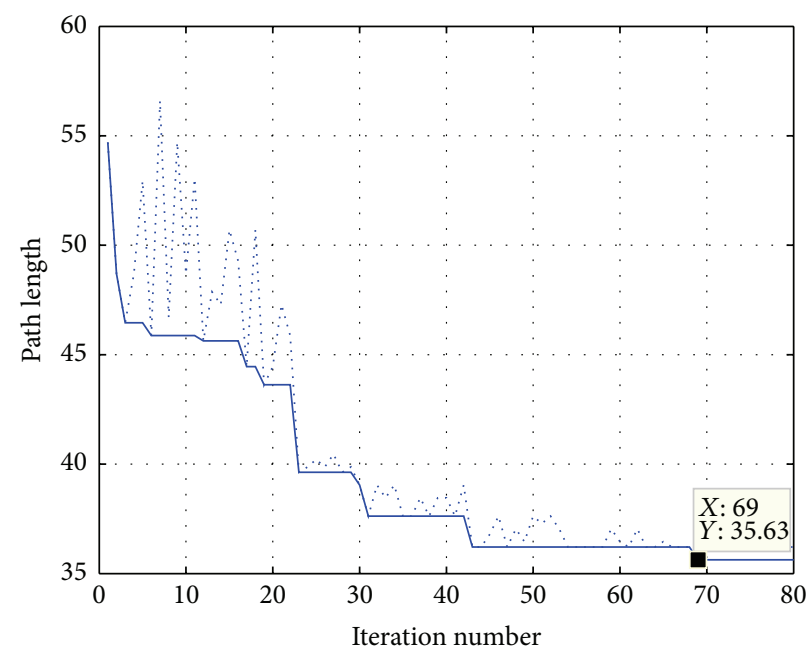

- The current global optimal path … The shortest path of each round

(b)

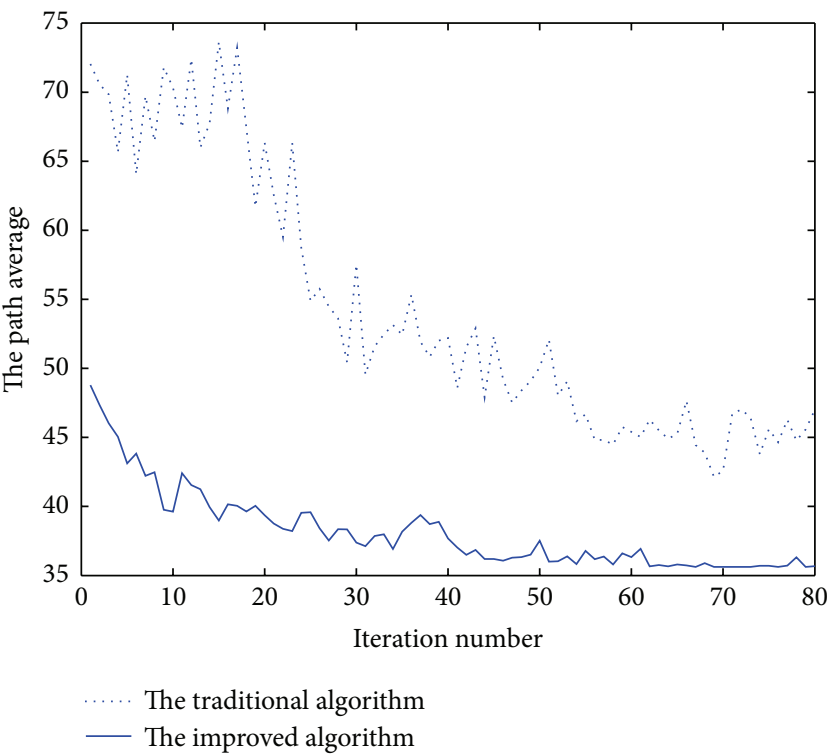

(d)

FIGURE 6: (a) Simulation results in $30 \times 30$ grids. (b) The iterative curve by the traditional algorithm. (c) The iterative curve by the improved algorithm. (d) The path average curve length by two algorithms.

the time efficiency and the simplicity of the algorithm and reduces the search space of ant colony algorithm. Adding the direction of the selection strategy can get more effective information as the heuristic information, more actively guide the search behavior of ants, and reduce the blindness. By using the rule of coverage, the search probability is reduced, the stability of the algorithm is guaranteed, and the effect of the quantity of the ants on the performance of the algorithm is guaranteed. The pheromone evaporation coefficient is segmented and adjusted, which can effectively balance the convergence speed and search ability of the algorithm.
Finally, it is strictly proven that the probability of finding an optimal solution is limited to 1 by the improved algorithm. The improved ant colony algorithm in solving the shortest path planning problem of the omnidirectional mobile vehicle has very good performance.

\section{Competing Interests}

The authors declare that there are no competing interests regarding the publication of this paper. 




(a)

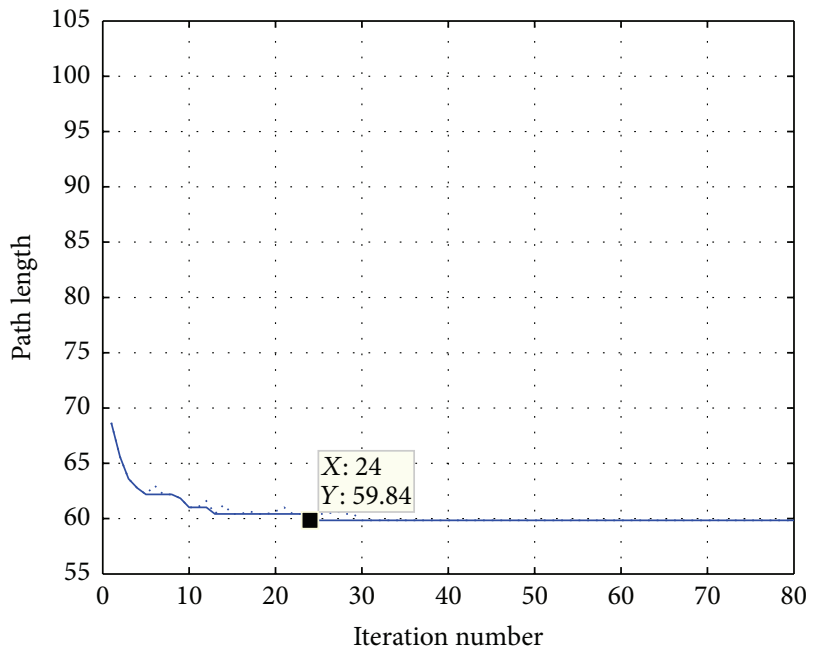

— The current global optimal path The shortest path of each round

(c)

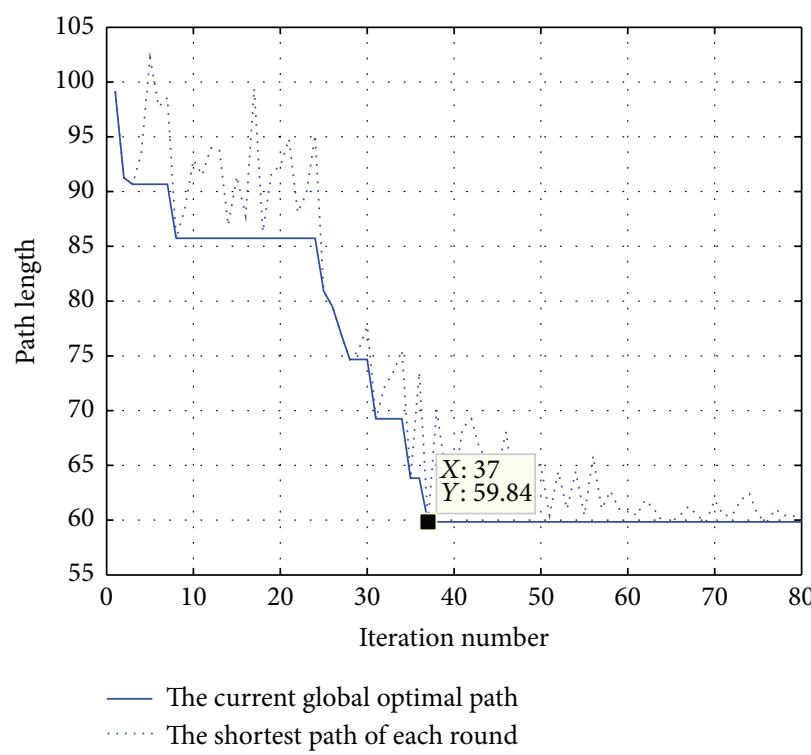

(b)



The traditional algorithm — The improved algorithm

(d)

FIgURE 7: (a) Simulation results in $40 \times 40$ grids. (b) The iterative curve by the traditional algorithm. (c) The iterative curve by the improved algorithm. (d) The path average curve length by two algorithms.

\section{Acknowledgments}

This research is supported by Science and Technology Research Project of Colleges and Universities in Hebei Province (Grant no. ZD2016142), Natural Science Foundation of Hebei Province (Grant no. F2014208013), and Doctoral Scientific Research Startup Foundation of Hebei University of Science and Technology (Grant no. QD201302).

\section{References}

[1] Y. Y. Gao, X. G. Ruan, H. J. Song, and J. J. Yu, "Path planning method for mobile robot based on a hybrid learning approach," Control and Decision, vol. 27, no. 12, pp. 1822-1827, 2012.
[2] T. Lozano-Perez and M. A. Wesley, "An algorithm for planning collision-free paths among polyedral obstacles," Communications of ACM, vol. 2, pp. 959-962, 1979.

[3] E. Masehian and D. Sedighizadeh, "Classic and heuristic approaches in robot motion planning-a chronological review," International Journal of Mechanical, Industrial Science and Engineering, vol. 1, no. 5, pp. 101-106, 2007.

[4] W. Lu, G. Zhang, and S. Ferrari, "An information potential approach to integrated sensor path planning and control," IEEE Transactions on Robotics, vol. 30, no. 4, pp. 919-934, 2014.

[5] J. Lee, B.-Y. Kang, and D.-W. Kim, "Fast genetic algorithm for robot path planning," Electronics Letters, vol. 49, no. 23, pp. 1449-1451, 2013. 
[6] Y. Fu, M. Ding, C. Zhou, and H. Hu, "Route planning for unmanned aerial vehicle (UAV) on the sea using hybrid differential evolution and quantum-behaved particle swarm optimization," IEEE Transactions on Systems, Man, and Cybernetics: Systems, vol. 43, no. 6, pp. 1451-1465, 2013.

[7] M. A. P. Garcia, O. Montiel, O. Castillo, R. Sepúlveda, and P. Melin, "Path planning for autonomous mobile robot navigation with ant colony optimization and fuzzy cost function evaluation," Applied Soft Computing, vol. 9, no. 3, pp. 1102-1110, 2009.

[8] H. Mo and L. Xu, "Research of biogeography particle swarm optimization for robot path planning," Neurocomputing, vol. 148, pp. 91-99, 2015.

[9] R.-E. Precup, E. M. Petriu, M.-B. Radac, E.-I. Voisan, and F. Dragan, "Adaptive charged system search approach to path planning for multiple mobile robots," in Proceedings of the 16th International Federation of Automatic Control, pp. 294-299, Sozopol, Bulgaria, September 2015.

[10] F. Ducho, A. Babineca, M. Kajana et al., "Path planning with modified a star algorithm for a mobile robot," Procedia Engineering, vol. 96, pp. 59-69, 2014.

[11] A. K. Guruji, H. Agarwal, and D. K. Parsediya, "Time-efficient $\mathrm{A}^{*}$ algorithm for robot path planning," Procedia Technology, vol. 23, pp. 144-149, 2016.

[12] A. Hidalgo-Paniagua, M. A. Vega-Rodríguez, and J. Ferruz, "Applying the MOVNS (multi-objective variable neighborhood search) algorithm to solve the path planning problem in mobile robotics," Expert Systems with Applications, vol. 58, pp. 20-35, 2016.

[13] A. T. Rashid, A. A. Ali, M. Frasca, and L. Fortuna, "Path planning with obstacle avoidance based on visibility binary tree algorithm," Robotics and Autonomous Systems, vol. 61, no. 12, pp. 1440-1449, 2013.

[14] M. Davoodi, F. Panahi, A. Mohades, and S. N. Hashemi, "Multi-objective path planning in discrete space," Applied Soft Computing, vol. 13, no. 1, pp. 709-720, 2013.

[15] A. Hidalgo-Paniagua, M. A. Vega-Rodríguez, J. Ferruz, and N. Pavón, "MOSFLA-MRPP: multi-objective shuffled frog-leaping algorithm applied to mobile robot path planning," Engineering Applications of Artificial Intelligence, vol. 44, no. 2342, pp. 123136, 2015.

[16] G.-F. Deng, X.-P. Zhang, and Y.-P. Liu, "Ant colony optimization and particle swarm optimization for robot-path planning in obstacle environment," Control Theory \& Applications, vol. 26, no. 8, pp. 879-883, 2009.

[17] J. He, Z. Tu, and Y. Niu, "A method of mobile robotic path planning based on integrating of GA and ACO," Computer Simulation, vol. 27, no. 3, pp. 170-174, 2010.

[18] P. K. Tiwari and D. P. Vidyarthi, "Improved auto control ant colony optimization using lazy ant approach for grid scheduling problem," Future Generation Computer Systems, vol. 60, pp. 7889, 2016.

[19] P. Wang, H.-T. Lin, and T.-S. Wang, "An improved ant colony system algorithm for solving the IP traceback problem," Information Sciences, vol. 326, pp. 172-187, 2016.

[20] W.-Y. Jiang, Y. Lin, M. Chen, and Y.-Y. Yu, "A co-evolutionary improved multi-ant colony optimization for ship multiple and branch pipe route design," Ocean Engineering, vol. 102, pp. 6370, 2015.

[21] M. Saidi-Mehrabad, S. Dehnavi-Arani, F. Evazabadian, and V. Mahmoodian, "An Ant Colony Algorithm (ACA) for solving the new integrated model of job shop scheduling and conflictfree routing of AGVs," Computers and Industrial Engineering, vol. 86, pp. 2-13, 2015.

[22] Z. Wang, X. Zhu, and Q. Han, "Mobile robot path planning based on parameter optimization ant colony algorithm," Procedia Engineering, vol. 15, pp. 2738-2741, 2011. 


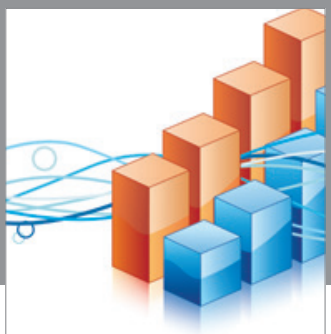

Advances in

Operations Research

vatem alat4

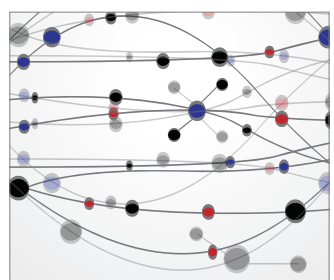

\section{The Scientific} World Journal
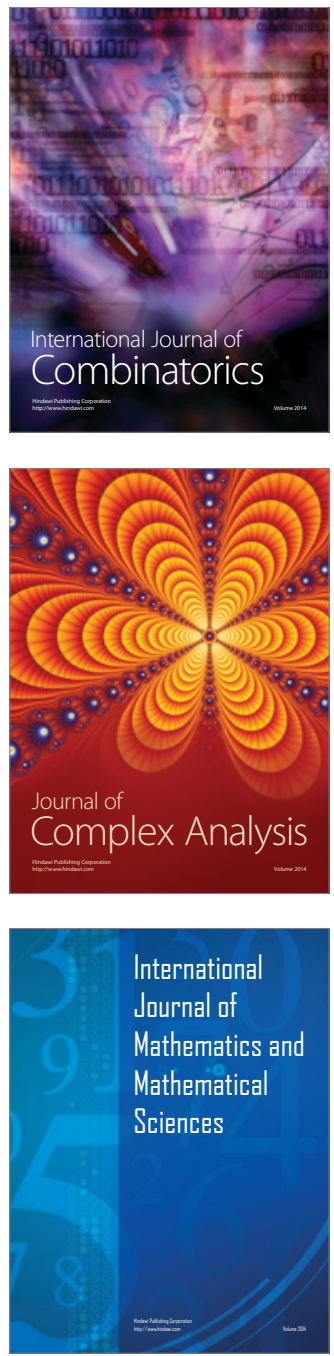


Algebra

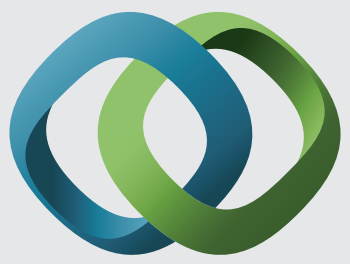

\section{Hindawi}

Submit your manuscripts at

http://www.hindawi.com
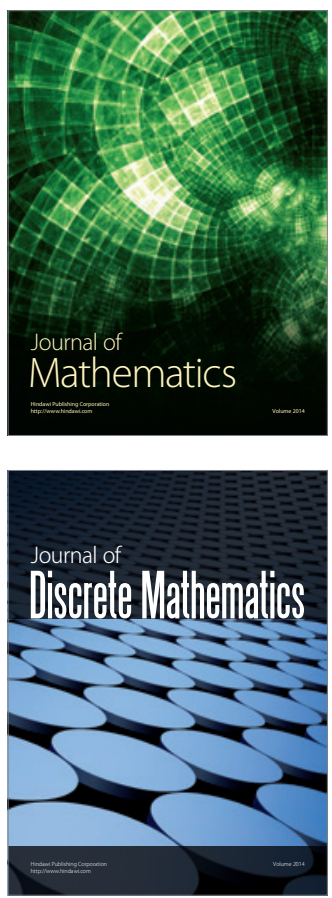

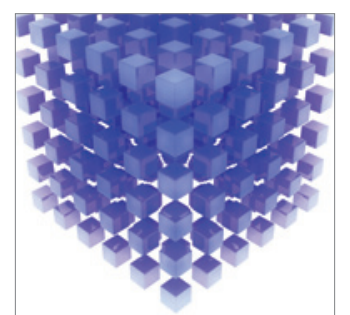

Mathematical Problems in Engineering
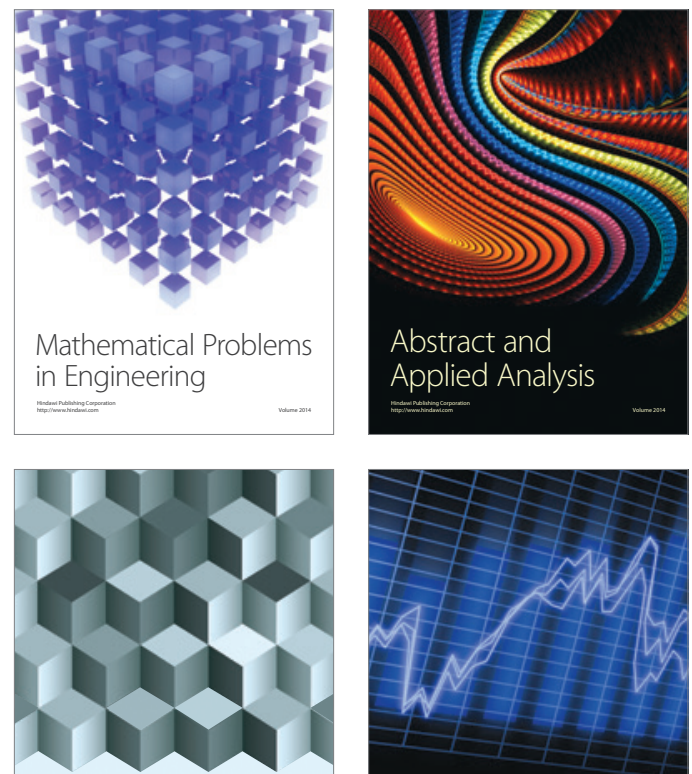

Journal of

Function Spaces

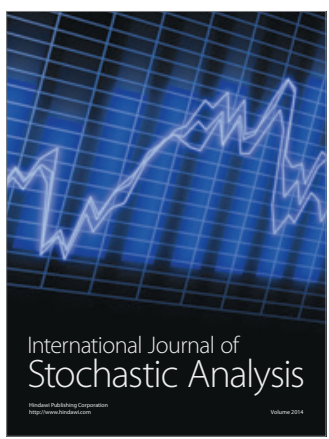


